\title{
Caesarean scar pregnancy: descriptive paper of three different types of management on a series of clinical cases
}

\author{
Ferdinando Antonio Gulino ${ }^{1}$, Elisa Pappalardo ${ }^{1}$, Carla Ettore $^{1}$, Antonio Simone Laganà ${ }^{2}$ \\ Stella Capriglione ${ }^{3}$, Giuseppe Ettore ${ }^{1}$ \\ ${ }^{1}$ Department of Obstetrics and Gynecology, Azienda di Rilievo Nazionale e di Alta Specializzazione (ARNAS) Garibaldi, Catania, Italy \\ 2Department of Obstetrics and Gynecology, "Filippo Del Ponte” Hospital, University of Insubria, Varese, Italy \\ ${ }^{3}$ Department of Obstetrics and Gynaecology, Campus Bio-Medico University, Rome, Italy
}

\begin{abstract}
Introduction: A caesarean scar pregnancy is a complex iatrogenic pathology, which represents a consequence of a previous caesarean section. It increased in recent years due to parallel increase of cesarean sections.

Material and methods: We present a retrospective study on patients with caesarean scar pregnancy diagnosed in our department from June 2016 to June 2019. Stable women with an embryo (with or without cardiac activity) who accepted our experimental protocol were treated with single dose of methotrexate (50 $\mathrm{mg}$ administered locally intracavitary $+50 \mathrm{mg}$ administered intramuscularly) and folinic acid (15 mg/day orally for 30 days). Clinically stable women with embryo (without cardiac activity) who decided to wait, were monitored by serial assays of $\beta$-hCG and clinical and ultrasonographic follow up. Women who were clinically unstable with embryo (without cardiac activity), were referred for urgent surgical treatment with dilation and curettage.

Results: Caesarean scar pregnancy was diagnosed in sixteen women. Among these women, seven were treated according to our experimental protocol with methotrexate and folinic acid and only one had profuse bleeding, which required a laparotomic hysterectomy. Four women were treated urgently with dilatation and curettage. Five women chose to wait: they were monitored and all spontaneously had a miscarriage.

Conclusions: In our preliminary study, we highlighted how our experimental protocol gave encouraging results in the first 10 weeks of caesarean scar pregnancy. However, caution is needed in patients with advanced gestational age, a gestational sac with large diameter, higher CRL and presence of embryonic cardiac activity.
\end{abstract}

Key words: caesarean scar pregnancy, methotrexate, expectant management, dilatation and curettage, miscarriage.

\section{Introduction}

A caesarean scar pregnancy is a complex iatrogenic pathology, which represents a consequence of a previous caesarean section. It is a condition difficult to treat, and could lead to complications during all three trimesters of pregnancy. It has had an exponential increase in recent years due to parallel increase of the number of cesarean sections.

Late consequences of caesarean sections, such as placenta praevia and placenta accreta spectrum disorders, are well known [1]; the long-term consequences are neither known nor documented. Clinically their expression in the first trimester is represented by the caesarean scar pregnancy. It is defined as a gestational sac, implanted in the scar area of a previous caesarean section.
Considering the increasing incidence of caesarean sections in recent years, the caesarean scar pregnancy has to be evaluated during the first trimester of pregnancy. The clinical management of a caesarean scar pregnancy is not easy because there are many options of treatment.

From an epidemiological point of view, the scientific literature described mainly case reports of caesarean scar pregnancy. Given the limited numbers of patients treated [1], it is difficult to define which is the best clinical practice for the management of this condition.

The most important scientific works on caesarean scar pregnancy were published in 2012 and 2015 by Timor-Tritsch et al. [1, 2]. However, despite this, no slandered treatment protocol has been universally accepted by the different scientific societies. The Royal College of Obstetricians and Gynaecologists (RCOG) guidelines 
defined the ultrasonographic criteria for diagnosing caesarean scar pregnancy on transvaginal scan; however, regarding the treatment, they assume that there is insufficient evidence to recommend any one specific intervention over another for caesarean scar pregnancy [3]. Therefore, it is also important to explain to the pa-

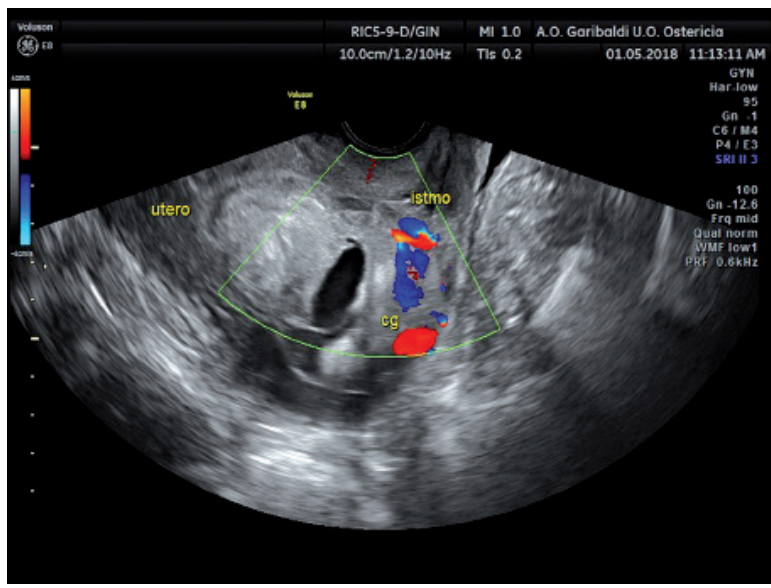

Fig. 1. Color flow of a scar pregnancy

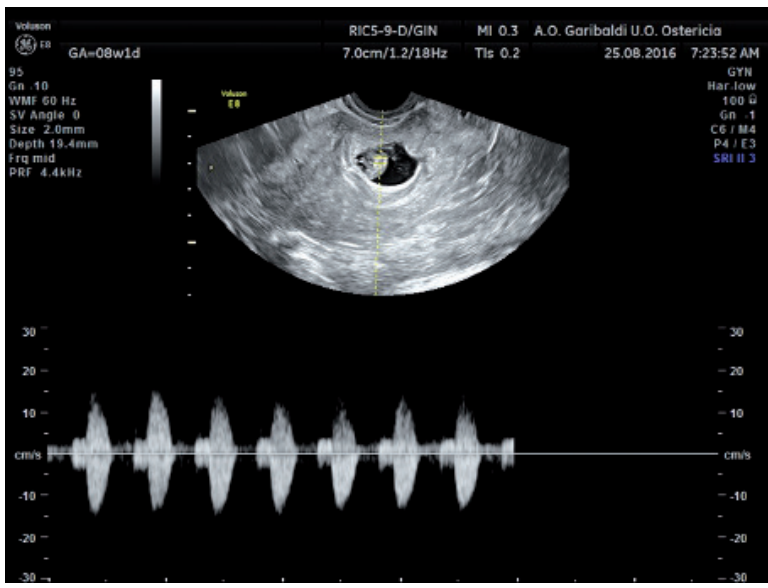

Fig. 2. Caesarean scar pregnancy with fetal activity

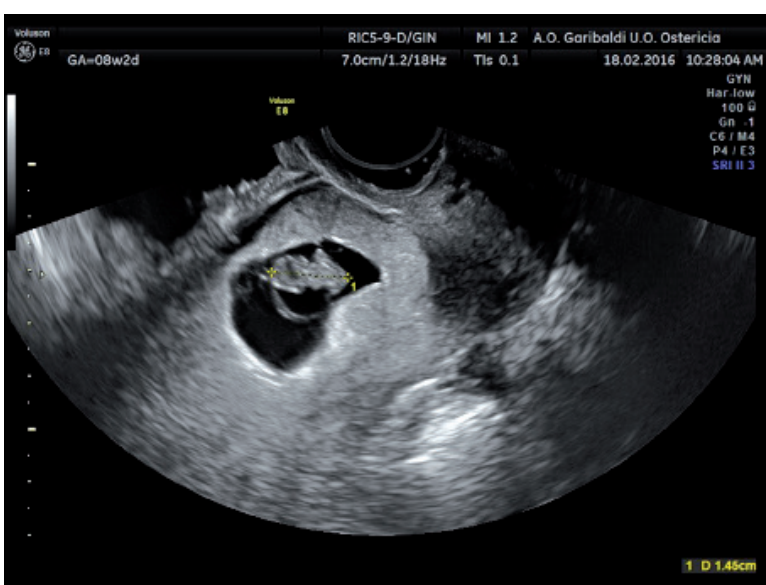

Fig. 3. Caesarean scar pregnancy and crown-rump length tient, by an accurate counseling, which are the different types of treatments of this clinical condition.

Aim of this study is the evaluation of the efficacy and safety of our experimental treatment protocol, based on the association between intracavitary and intramuscular methotrexate, for patients affected by caesarean scar pregnancy, over a period of 3 years, between June 2016 and June 2019.

\section{Material and methods}

This was a retrospective experimental study conducted in a tertiary referral center of Obstetrics and Gynecology, the "Azienda di Rilievo Nazionale e di Alta Specializzazione" (ARNAS) Garibaldi Nesima of Catania (Italy). All women with a caesarean scar pregnancy, diagnosed in the period between June 2016 and June 2019, were included in the study. This analysis was performed considering the medical records of the first aid department.

The diagnosis of caesarean scar pregnancy was performed by transvaginal ultrasound with an IC5-9-D endocavitary probe of a GE Voluson E8 Expert BT13 ultrasound, with the ultrasound criteria described by Timor-Tritsch et al. [2]:

1) empty uterus,

2) empty endocervical canal,

3) thin or absent layer of myometrium between gestational sac and urinary bladder,

4) gestational sac or trophoblast sited anteriorly, at the level of internal os, or lower uterine segment at the site of the previous hysterotomy scar,

5) evidence of trophoblastic/placental blood flow on Doppler examination.

Caesarean scar pregnancy was diagnosed in sixteen women between June 2016 and June 2019. Some examples were shown in Figures 1-3.

Medical management with high doses of methotrexate and folinic acid (15 mg/day orally for 30 days) as first-line therapy was offered to all clinically stable women and embryo with or without cardiac activity, but without evidence of hemorrhage, or suspected uterine rupture, as assessed ultrasonographically. Liver and kidney function tests, and patient reliability for follow-up was also assessed. There were no exclusion criteria due to gestational age, $\beta$-hCG level and diameter of the gestational sac. These women were treated according to our experimental protocol with single dose of methotrexate (50 mg administered locally intracavitary + $50 \mathrm{mg}$ administered intramuscularly) and folinic acid (15 mg/day orally for 30 days). During this treatment, an antibiotic prophylaxis with clarithromycin per os (500 mg $\times 2$ for 5 days) or intramuscular cefazolin ( $1 \mathrm{~g} \times 2$ for 5 days) was administrated.

The patients who underwent to this type of treatment, according to Italian law, signed an informed con- 
Table 1. Results of the clinical study

\begin{tabular}{|c|c|c|c|c|c|c|c|c|c|c|c|}
\hline $\begin{array}{l}\text { Clinical } \\
\text { cases }\end{array}$ & Age & $\begin{array}{l}\text { No. of } \\
\text { previous } \\
\text { CS }\end{array}$ & $\begin{array}{l}\text { Gestational } \\
\text { age }\end{array}$ & $\begin{array}{l}\text { Basal } \\
\beta \text {-hCG }\end{array}$ & $\begin{array}{l}\text { Fetal } \\
\text { heart }\end{array}$ & $\begin{array}{l}\text { Success } \\
\text { of MTX } \\
\text { protocol }\end{array}$ & $\begin{array}{c}48-72 \text { h } \\
\beta-h C G\end{array}$ & $\begin{array}{l}5 \text { days } \\
\beta \text { hcg }\end{array}$ & MSD & CRL & $\begin{array}{c}\text { Other } \\
\text { interventions }\end{array}$ \\
\hline 1 & 30 & 1 & $6+1$ & 26150 & $\sqrt{ }$ & $\sqrt{ }$ & 30482 & 28654 & $18 \mathrm{~mm}$ & 5.6 & \\
\hline 2 & 37 & 3 & $8+0$ & 62458 & $\sqrt{ }$ & $\sqrt{ }$ & 61966 & 37068 & $20 \mathrm{~mm}$ & 7.2 & \\
\hline 3 & 26 & 3 & $8+3$ & 30355 & $x$ & $\sqrt{ }$ & 29794 & 1 & $17 \mathrm{~mm}$ & 1 & \\
\hline 4 & 47 & 3 & ND & 6557 & $x$ & $\sqrt{ }$ & 1124 & 1 & $17 \mathrm{~mm}$ & I & \\
\hline 5 & 24 & 3 & $7+3$ & 222123 & $\sqrt{ }$ & $\sqrt{ }$ & 171623 & 51506 & $14 \mathrm{~mm}$ & 7.0 & \\
\hline 6 & 38 & 1 & $7+0$ & 70923 & $\sqrt{ }$ & $\sqrt{ }$ & 49441 & 1 & $19 \mathrm{~mm}$ & 9.4 & \\
\hline 7 & 35 & 2 & $11+0$ & 81181 & $\sqrt{ }$ & $x$ & 51418 & 42447 & $41 \mathrm{~mm}$ & 15 & Hysterectomy \\
\hline 8 & 32 & 1 & $8+1$ & 47520 & $x$ & 1 & 1 & 1 & $18 \mathrm{~mm}$ & 7 & $D \& C$ \\
\hline 9 & 43 & 2 & $6+2$ & 13476 & $x$ & I & I & I & $12 \mathrm{~mm}$ & 4.7 & $D \& C$ \\
\hline 10 & 38 & 2 & $6+6$ & 19836 & $x$ & I & I & I & $16 \mathrm{~mm}$ & I & $D \& C$ \\
\hline 11 & 42 & 1 & $7+3$ & 22941 & $x$ & I & I & I & $16 \mathrm{~mm}$ & I & $D \& C$ \\
\hline 12 & 40 & 3 & $6+0$ & 9250 & $x$ & 1 & 1 & 1 & $14 \mathrm{~mm}$ & 4 & \\
\hline 13 & 35 & 2 & $6+2$ & 13654 & $x$ & I & I & I & $16 \mathrm{~mm}$ & 5.2 & \\
\hline 14 & 36 & 2 & $5+6$ & 3146 & $x$ & I & I & 1 & $10 \mathrm{~mm}$ & I & \\
\hline 15 & 43 & 2 & $6+0$ & 4810 & $x$ & 1 & I & I & $12 \mathrm{~mm}$ & I & \\
\hline 16 & 42 & 1 & $6+5$ & 5220 & $x$ & 1 & I & 1 & $14 \mathrm{~mm}$ & 1 & \\
\hline
\end{tabular}

MSD - mean sac diameter, CRL - crown-rump lenght, MTX - metotrexate, CS - caesarean section

sent for abortion according to the law 194/78 art. 5 . The patients were not hospitalized because they were clinically stable, but they were advised to come into our hospital if there was abdominal pain or bleeding. The patients were adequately informed about the proposed type of treatment and the risks related to scar pregnancy.

A waiting procedure, with serial assays of $\beta$-hCG and clinical and ultrasound monitoring was proposed to all clinically stable patients with embryo devoid of cardiac activity, without evidence of hemorrhage, and no suspicion of uterine rupture, as assessed ultrasonographically, with normal hepatic and renal function and patient reliability for follow-up. The serial assays of $\beta$-hCG were performed at the first clinical examination, after 48-72 h, and after 7 days.

Women who were clinically unstable with embryo devoid of cardiac activity, with evidence of menometrorrhagia, or suspected uterine rupture assessed by ultrasound, were referred for urgent surgical treatment with dilation and curettage, and subsequent close monitoring of vital signs and blood loss.

Following data were collected: maternal age, number of previous caesarean sections, gestational age, mean gestational sac diameter, crown-rump length $(\mathrm{CRL})$, presence of embryonic cardiac activity, initial and subsequent $\beta$-hCG levels, methotrexate side effects, treatment outcome. The correct therapy with metho- trexate was confirmed by subsequent re-evaluations of serum $\beta$-hCG level $(<5 \mathrm{IU} / \mathrm{l})$.

Data were analyzed using SPSS version 23 (IBM Corp, Armonk, NY). The study was approved by the institutional ethics committee for human research of the ARNAS Garibaldi.

\section{Results}

Caesarean scar pregnancy was diagnosed in sixteen women between June 2016 and June 2019 . Among these women, seven were treated according to our experimental protocol with single dose of methotrexate $(50 \mathrm{mg}$ administered locally intracavitary + $50 \mathrm{mg}$ administered intramuscularly) and folinic acid (15 mg/day orally for 30 days). Four women, given the presence of high blood loss, were treated urgently with instrumental revision of the uterine cavity (dilation and curettage). Five women, on the other hand, chose to wait; they were monitored and all of them had spontaneous miscarriage (Table 1). Their age ranged from 24 to 47 years, and the number of previous caesarean sections ranged from 1 to 3 . Gestational age ranged from $5+6$ to $11+0$ weeks based on their last menstrual cycle. The diameter of the gestational sac ranged from $10 \mathrm{~mm}$ to $41 \mathrm{~mm}$ and the CRL (identified in nine women) ranged from $4 \mathrm{~mm}$ to $15 \mathrm{~mm}$. Embryonic cardiac 
activity was found in five women. The baseline $\beta$-hCG level ranged from 3146 to $222123 \mathrm{IU} / \mathrm{l}$.

There were no adverse effects related to methotrexate, such as bone marrow suppression, lung fibrosis, nonspecific pneumonia, liver cirrhosis, renal failure and gastric ulcer. None of the women under examination complained of nausea. Out of seven women who were treated with methotrexate, only one had profuse bleeding, which required a laparotomic hysterectomy. This patient disappointed our serial follow up and she was in an advanced stage of pregnancy, at 11 weeks of gestational age.

Three women were lost to the serial $\beta$-hCG test. However, none of the women who referred to our clinic came back complaining of specific clinical signs or symptoms. Patients who were treated urgently by dilatation and curettage did not have any clinical problem.

The patients who chose a waiting management had miscarriages, and there were no signs of previous scar pregnancy on ultrasound examination done at the follow up.

\section{Discussion}

In our work we have decided to perform an experimental protocol with single dose of methotrexate (50 mg administered locally intracavitary $+50 \mathrm{mg}$ administered intramuscularly) and folinic acid (15 mg/day orally for 30 days) which needs a close monitoring.

In 2015 a systematic review on the efficacy of systemic methotrexate for caesarean scar pregnancy treatment identified 40 cases from 27 articles, reporting a $55 \%$ success rate ( 22 out of 40 ) with systemic methotrexate alone, and a $85 \%$ success rate (34 of 40 cases) with further minor interventions such as methotrexate locally injected, dilatation and curettage, or dilatation and curettage and embolization of the uterine arteries [4]. In 2017 another systematic review reported a 56\% success rate for systemic methotrexate therapy alone [5]. In literature, methotrexate is usually given as a single therapy [6-8] or in multiple-dose regime [9-10]. The single-dose regimen included a dosage of $50 \mathrm{mg} / \mathrm{m}^{2}$ (body surface) [6-8]. Dosage would be $80 \mathrm{mg}$ for an average body surface of $1.6 \mathrm{~m}^{2}$. In multiple doses a regimen of $50 \mathrm{mg} / \mathrm{m}^{2}$ (body surface) [10] or $1.0 \mathrm{mg} / \mathrm{kg}$ (weight) [9] of intramuscular methotrexate was administered every other day for about 8-10 doses, so the total amount of methotrexate would be higher, but given over a longer period. Patients were given calcium foliate for detoxication $12 \mathrm{~h}$ after the injection [10].

In the study by Tanaka et al. [11], 24 out of 28 women $(85.7 \%)$ with high dose intravenous methotrexate therapy alone and $15 \mathrm{mg}$ folinic acid (leucovorin) orally given at 30, 42, 54 and $66 \mathrm{~h}$ post commencement of methotrexate, were treated successfully; 3 women $(10.7 \%)$ required suction evacuation following initial treatment with methotrexate and folinic acid. This study also demonstrated that the efficacy of high dose intravenous methotrexate therapy for caesarean scar pregnancy (85.7\%, 24 out of 28 women) is similar to its efficacy for interstitial ectopic pregnancy $(93.9 \%, 31$ of 33 women).

Some authors argued instead that systemic methotrexate may not be effective in the treatment of caesarean scar pregnancy due to the surrounding fibrous scar tissue, rather than normally vascular myometrium [12].

In our scientific work, we have chosen this protocol because some other scientific works [8-11] have demonstrated clinical efficacy of higher doses of methotrexate. We have chosen this higher dose of folinic acid to avoid any toxicity related to the higher single dose of methotrexate.

The data of our study, although interesting and promising, have to be considered preliminary, related to the smallness of our sample. Further studies with larger sample of patients could give more information about this pathological condition.

\section{Conclusions}

In our preliminary study, we highlighted how the treatment with methotrexate injected in the gestational sac, in association with methotrexate i.m. gave encouraging results in the first 10 weeks of caesarean scar pregnancy.

However, caution is needed in patients with advanced gestational age ( $>10$ weeks), or when the ultrasound shows a gestational sac with large diameter (> $30 \mathrm{~mm}$ ), higher CRL (>12 $\mathrm{mm}$ ) and presence of embryonic cardiac activity. In these cases, additional therapy may be necessary. A primary obstetric objective must therefore be a diagnosis as early as possible to avoid subsequent complications.

\section{Disclosure}

The authors report no conflict of interest

\section{References}

1. Timor-Tritsch IE, Khatib N, Monteagudo A, et al. Cesarean Scar Pregnancies. J Ultrasound Med 2015; 34: 601-610.

2. Timor-Tritsch IE, Monteagudo A, Santos R, et al. The diagnosis, treatment, and follow-up of cesarean scar pregnancy. Am J Obstet Gynecol 2012; 207: 44e1-e13.

3. Elson CJ, Salim R, Potdar N, et al. on behalf of the Royal College of Obstetricians and Gynaecologists. Diagnosis and management of ectopic pregnancy. BJOG 2016; 123: e15-e55.

4. Bodur S, Özdamar O, Kiliç S, Gün I. The efficacy of the systemic methotrexate treatment in caesarean scar ectopic pregnancy: A quantitative review of English literature. J Obstet Gynaecol 2015; 35: 290-296.

5. Maheux-Lacroix S, Li F, Bujold E, et al. Cesarean Scar Pregnancies: A Systematic Review of Treatment Options. J Minim Invasive Gynecol 2017; 21: 915-925. 
6. Lian F, Wang Y, Chen W, et al. Uterine artery embolization combined with local methotrexate and systemic methotrexate for treatment of cesarean scar pregnancy with different ultrasonographic pattern. Cardiovasc Intervent Radiol 2012; 35: 286-291.

7. Yang XY, Yu H, Li KM, et al. Uterine artery embolisation combined with local methotrexate for treatment of caesarean scar pregnancy. BJOG 2010; 117: 990-996.

8. Peng P, Ggui T, Liu X, et al. Comparative efficacy and safety of local and systemic methotrexate injection in cesarean scar pregnancy. Ther Clin Risk Manag 2015; 11: 137-142.

9. Kutuk MS, Uysal G, Dolanbay M, Ozgun MT. Successful medical treatment of cesarean scar ectopic pregnancies with systemic multidose methotrexate: Single-center experience. J Obstet Gynaecol Res 2014; 40: 1700-1706

10. Yang H, Li S, Ma Z, Jia Y. Therapeutic effects of uterine artery embolisation (UAE) and methotrexate (MTX) conservative therapy used in treatment of cesarean scar pregnancy. Arch Gynecol Obstet 2016; 293: 819-823.

11. Tanaka K, Coghill E, Ballard E, et al. Management of caesarean scar pregnancy with high dose intravenous methotrexate infusion therapy: 10-year experience at a single tertiary centre. Eur J Obstet Gynecol Reprod Biol 2019; 237: 28-32.

12. Ravhon A, Ben-Chetrit A, Rabinowitz R, et al. Successful methotrexate treatment of a viable pregnancy within a thin uterine scar. BJOG AnInt J Obstet Gynaecol. 1997; 104: 628-629. 